\title{
XXVIII.
}

Aus der medicinisehen Klinik zu Königsberg i. Pr.

\section{Ueber die Bildungsstätte des Gallenfarbstoffs beim Kaltblüter.}

\author{
Von \\ Dr. Valentini, \\ Assistenzarzt der medicinischen Klinik.
}

Die seit Virchow allmählich erworbene Erkenntniss, dass in alten Blutextravasaten das Hämoglobin sich in Hämatoidin zersetzt und letzteres mit dem Biliverdin identisch ist, machte es im Verein mit der Beobachtung, dass bei vermehrtem Zerfall von rothen Blutkörperchen und dadurch bedingtem Freiwerden von Hämoglobin in der Blutbahn eine vermehrte Gallenfarbstoff bildung eintritt, höchst wahrseheinlich, dass der Gallenfarbstoff ein Abkömmling des Blutfarbstoff sei. Viel schwieriger erwies es sich, über die Bildungsstätte desselben Sicherheit zu erlangen.

Wäbrend die Einen die Leber als Excretionsorgan für den im Blut präformirten Gallenfarbstoff ansahen, nahmen Andere an, dass derselbe erst in den Leberzellen gebildet wird.

Der Gedanke, durch eine Entleberung von Thieren hiertiber Klarheit zu erlangen, liegt nahe und ist schon von J.Müller, Moleschott und $K u n d e$ beim Froseh zur Ausfïhrung gebracht. In neuester Zeit nahm Stern ') auf $\mathrm{N}$ a u n yn's Anregung diese Versuche wieder auf. Er konnte bei entleberten Fröschen, die 2-8 Tage lebten, weder in den Organen noch in den Flüssigkeiten des Körpers Gallenfarbstoff constatiren. Diese Versuche verloren jedoch völlig ihre Beweiskraft, als sich nach Unterbindung des Ductus choledochus ergab, dass auch bei erbaltener Leber bei Fröschen keine Anbäufung von Gallenfarbstoff im Organismus eintritt. Während so der Frosch zu Experimenten iuber den Icterus unbrauchbar ersehien, war die Entleberung von Säugethieren wegen schnell tödtlich endender Circulationsstörungen im Gebiet der Vena portarum stets missglückt.

1) Ueber die normale Bildungsstätte des Gallenfarbstoffs. Dies. Arch. XIX. Bd. 
Einen besseren Erfolg ergaben Versuche an Vögeln, bei denen ausgedehnte Collateralen zwischen der Nierenvene und der Vena portarum tödtliche Stauungen im Gebiet der Leberpfortader verhindern konnten. Es gelang Stern, bei Tauben die Leber aus dem Kreislauf auszuschalten und die Abwesenheit von Gallenfarbstoff, der in einer anderen Versuchsreihe nach Verschluss des Ductus choledochus im Blut und den Organen constatirt war, bei einer durchschnittlich 22 stündigen Beobachtungsdauer festzustellen.

Naunyn ${ }^{1}$ ) und Minkowski wiederholten bei Gänsen, Enten und Hühnern diese Versuche und kamen zu gleichem Resultat. Das Auftreten einer geringen Menge Gallenfarbstoff im Urin entleberter Thiere erklärten sie theils aus den technischen Schwierigkeiten der Operation, indem entweder kleine functionirende Leberstïckchen in der Bauchböhle zurtickblieben, oder Galle bei der Exstirpation in das Peritoneum floss, theils aus der Resorption vom Darme aus, der bei Gänsen grosse Mengen Biliverdin enthält. Dieser letzteren Erklärung steht nichts entgegen, seit Sehiff die Resorption von Galle aus dem Darm wahrscheinlich gemacht hat, und erscheint besonders einleuchtend, wenn man in Betracht zieht, dass die Thiere nach der Entleberung viel Wasser za sich nahmen und viel Urin ausschieden, so dass die günstigsten Bedingungen für die Resorption des in reichen Wassermengen gelösten Biliverdin vorhanden waren. - Ferner suchten sie durch Arsenwasserstoffvergiftung bei entleberten Thieren weitere Beweise fïr Gallenfarbstoffbildung in der Leber zu finden.

Bekanntlich folgt der Einathmung von Arsenwasserstoff ein Freiwerden des Hämoglobin aus den rothen Blutkörperchen, dann ein Icterus und bei starker Vergiftung eine Hämoglobinurie. Wenn bei entleberten Thieren, die mit $\mathrm{AsH}_{3}$ vergiftet waren, kein Biliverdin im Harn auftrat, sondern nur Hämoglobin, so war bewiesen, dass die Zersetzung des Blutfarbstoff in einer Weise, die zur Entstehung eines Icterus führen kann, nur in der Leber erfolgt. Sie vergifteten also Vögel mit Arsenwasserstoff und fügten, sobald durch Auftreten von Biliverdin im Urin die Wirksamkeit der Vergiftung sicher war, eine Entleberung hinzu. Der Urin wurde fast gallenfrei und eine starke Hämoglobinurie trat an seine Stelle.

Es blieb äbrig, die Abwicklung des Processes in der Leber zu verfolgen. Zn diesem Zweck wurde durch Arsenwasserstoffvergiftung eine vermehrte Gallenbildung erzeugt und die Leber mikroskopisch untersucht. Dabei zeigten sich schon $11 / 2$ Stunden nach der Ver-

1) Beiträge zur Pathologie der Leber und des Icterus. Dies. Arch. XXI. Bd. Arehiv f. experiment. Pathol. u. Pharmakol. XxIv. Bd. 
giftung vereinzelt blutkörperchenhaltige Zellen in den Lebercapillaren, deren Menge allmählich zunahm und nach 3-4 Stunden eine sehr erhebliche war. $\mathrm{Zu}$ dieser Zeit traten dann Zellen von gleicher Grösse wie die vorigen auf, in denen entweder mit rothen Blutkörperchen oder allein rostfarbene Partikel ${ }^{1}$ ) lagen, die durch die Behandlung mit.Ferrocyankalium und Salzsäure sich blau färbten und somit eisenhaltig waren. Es ist das Auftreten dieser Eisenreaction das erste Zeichen der Zersetzung des Blutfarbstoffs, und man darf annehmen, dass in diesen blutkörperchenhaltigen Zellen aus der Zersetzung des Blutfarbstoffs im weiteren Verlauf Gallenfarbstoff hervorgeht. Denn Naunyn und Minkowski sahen nach Anwendung der Sublimathärtung in diesen gleichen blutkörperchenhaltigen Zellen in der Leber Gallenfarbstoffpartikelchen, welche nach Allem dort entstanden waren. In der Milz and im Knochenmark worden zwar blutkörperchen- und eisenhaltige Zellen, niemals aber gallenfarbstoff haltige gefunden.

Gleichzeitig mit diesen Vorgängen an den weissen Blutkörperchen trat eisenhaltiges Pigment in den Leberzellen auf. Anfangs diffus in den Zellen vertheilt, wurde es schnell nach dem Pol der Zelle, der dem Gallengang zugekehrt war, befördert, so dass es auf Quersehnitten ringförmig, auf Längsschnitten reihenweise denselben umgab.

Was die Deutung dieses Befundes betrifft, so ist es klar, dass das eisentaltige Pigment der weissen Blutkörperchen und Leberzellen als Zersetzungsproduct des Hämoglobin aufzufassen ist. In den Leberzellen liess sich von Gallenfarbstoff nichts nachweisen, doch schien die Anwesenheit von Eisen dafür zu sprechen, dass hier eine Zersetzung von Hämoglobin stattfand. Andererseits war es auch möglich, dass das in den Leberzellen nachweisbare Eisen bereits an anderer Stelle, z. B. im Blute abgespalten war und dass seine Anwesenheit in den Leberzellen nur auf die Ausscheidung des Eisens durch die Leber zu beziehen sei. Zwar war die Annahme, dass die Gallenfarbstoffbildung in dem circulirenden Blute statthabe, durch die Ergebnisse der Entleberung bei mit Arsenwasserstoff vergifteten Thieren höchst unwahrscheinlich gemacht, doch gab die Thatsache, dass sich bei

1) Uebrigens ist es in keiner Weise nachgewiesen, dass es sich bei diesen rostfarbenen Partikelchen, welche die Eisenreaction geben, um ein besonderes, aus dem Hämoglobin entstandenes Pigment handelt. Vielmehr sind die rostfarbenen Partikelchen wahrscheinlich nichts Anderes als Eiweiss-(Globulin-)Reste des Hämoglobin oder die Stromata der rothen Blutkörper, welche durch Eisenoxỳdalbuminat oder vielleicht durch Reste von Hämatin gefärbt sind. 
den Vögeln in den blutkörperchenhaltigen Zellen Gallenfarbstoff bildet, immerhin einem Zweifel Raum. Zugegeben werden muss, dass möglicherweise beim normalen Thier ohne Leberexstirpation sich deshalb mehr Galle bilde, weil die blutkörperchenhaltigen Zellen der Leber, nicht weil die Leberzellen fehlten; wenn auch die von Naunyn und Minkowski erhobene Beobachtung, dass die blutkörperchenhaltigen Zellen in der Leber in vielen Fällen von Icterus nach der Arsenwasserstoffvergiftung sehr spärlich waren, entschieden dagegen sprach.

Auf den Rath des Herrn Prof. Naun yn versuchte ich die Lösung dieser Frage nochmals beim Kaltblitter in Angriff zu nehmen. Die Langsamkeit, mit der Stoffwechselvorgänge und Zellthätigkeit sich bei ihnen vollziehen, liess hoffen, dass die blutkörperchenhaltigen Zellen hier keine Rolle spielen werden, oder dass vielleicht ihre Rolle bei der Gallenfarbstoff bildung zeitlich von der der Leberzellen getrennt sei und daher besser wie bei den Vögeln mit ihrem enorm schnellen Stoffwechsel übersehen werden könne.

Da der Frosch, das gewöhnlich zu Versuchen an Kaltblïtern benutzte Thier, nach den von Stern mitgetheilten Versuchen hierzu nicht geeignet schien, habe ich mich kleiner Wasserschildkröten im Durchschnittsgewicht von $60-70 . \mathrm{g}$ bedient. Ihr Stoffwechsel scheint etwas reger als der des Frosches zu sein, und allen schädlichen Eingriffen gegenüber entfalten sie eine bedeutende Lebenskraft.

Dieselben wurden der Einwirkung von verdünntem Arsenwasserstoff, der aus einer Lösung von arsenigsaurem Kali mit Zink und Salzsäure entwickelt wurde, während 10-15 Minuten ausgesetzt. Nach der Vergiftung waren sie völlig munter und verhielten sich wie gesunde Thiere. Am nächsten Tage waren sie dann ersichtlich matter, krochen wenig umber und reagirten nur langsam auf mechanische Reize. Einige starben schon im Verlauf des nächsten Tages. Der grösste Theil wurde dann in den nächsten acht Tagen getödtet. Drei wurden übrig gelassen und gingen erst nach 7 Wochen zu Grunde. Bei der Untersuchung der Thiere richtete sich das Hauptaugenmerk auf den Urin, Leber, Milz und Niere. Letztere wurden mikroskopisch nach blutkörperchen- und eisenhaltigen Zellen durchsucht und auf das Auftreten von Eisen in ihren Organbestandtheilen geachtet. $\mathrm{Zu}$ diesem Zweck wurden theils frische Zupfpräparate benutzt, theils Schnitte, die in Alkohol oder in Sublimatlösung nach Naunyn und Minkowski gehärtet waren. Auf ihren Eisengehalt wurden die Alkoholpräparate nach der von Perls angegebenen Methode mit Ferrocyankalium und Salzsäure, wobei eisenhaltige Körper sich blau 
färben, geprïft. Die von Quincke angegebene Methode mit Schwefelammonium war nicht zweckmässig, da sich in der Leber von Schildkröten viel sehwarzes Pigment findet, das bei der Quincke'schen Eisenreaction von den durch Schwefelammonium schwarzgefärbten Körpern schwer zu unterscheiden ist. Mit Ferrocyankalium und Salzsäure färbt sich dieses Pigment nicht blau. Bemerkt sei noch, dass ich bei der Herstellung der Präparate stets Glasnadeln anwandte.

Bevor ich nun ïber die Ergebnisse der Arsenwasserstoffvergiftung: bei Schildkröten berichte, scheint es zweckmässig, auf das Verhalten der Excrete und Organe normaler Schildkröten, soweit sie bei diesen Untersuchnngen in Betracht kommen, einzugehen. Der Urin, der durch Katheterisation vermittelst einer capillaren Glasröhre gewonnen wurde, war in allen Fällen wasserklar. Die Leber ist von braunrother Farbe. Nach Behandlung von Leberschnitten mit Ferrocyankalium und Salzsäure tritt entweder gar keine Veränderung ein, oder der Schnitt nimmt einen leicht grünlich-blauen Farbenton an, der unter dem Mikroskop bei schwacher Vergrösserung demselben ein grünliches Colorit giebt, während bei starker Vergrösserung nichts davon wahrzunehmen ist und eine Unterscheidung zwischen einem normalen und auf Eisen geprüften Schnitt nicht zu finden ist. In der Niere und Milz ist niemals eine Spur von eisenhaltigen Körpern nachzuweisen. Das absolute Fehlen von eisenhaltigen Körpern in den oben genannten Organen normaler Schildkröten gestattet eine sichere Beurtheilung abnorm vorhandenen Eisens und unterscheidet sie für diese Versuche vortheilhaft vom Warmblüter, bei dem Quincke ${ }^{1}$ ) einen in ziemlich weiten Grenzen schwankenden Eisengehalt der Leber, Milz und des Knochenmarks fand, so dass es ihm oft schwierig war, eine abnorme Vermehrung ihres Eisengehaltes sicher festzustellen.

Die Ergebnisse der Arsenwasserstoffvergiftung bei Schildkröten sind nun folgende:

Am dritten bis vierten Tage, in einem Falle sogar schon am zweiten, konnte eine Grünfärbung des bis dahin wasserklaren Urins constatirt werden. Dieselbe hielt bis zum Tode des Thieres an und nahm allmählich an Intensität zu. Die Leber zeigte sich bei denen, die bald nach dem Auftreten des Icterus getödtet waren, leicht grünlich verfärbt. Einige lebten bei stets zunehmendem Biliverdingehalt des Urins erstaunlich lange. Sie starben erst nach 7 Wochen und zeigten entsprechend dem stark ikterischen Urin eine intensive Grünfärbung der Leber und des subcatanen Bindegewebes, während an

1) Ueber Siderosis. II. Deutsch. Arch. f. klin. Med. XXVII. Bd. 
der Niere und Milz makroskopisch nichts Abnormes wahrzunehmen war. Die mikroskopische Untersuchung der Leber nach Härtung in Sublimat zeigte bei diesen Thieren eine Anhänfung von Gallenfarbstoff in ihren Zellen. Derselbe war aber nicht gleichmässig in ihnen vertheilt, sondern an dem Ende der Zelle, das dem Gallengang zugekehrt war, aufgehäuft. Dies konnte vorzugsweise an Querschnitten von Gallengängen sichergestellt werden. In der Niere waren einzelne Harnkanälchen mit einer grünen Masse erfüllt, die Glomeruli und Gefässe waren frei davon.

Das Auftreten von Icterus bei der Schildkröte ist insofern von Interesse, als es bis jetzt noch nicht gelungen ist, bei Kaltblïtern denselben zu erzeugen.

Im Ganzen habe ich die Leber and Milz von 20 mit Arsenwasserstoff vergifteten Schildkröten untersucht. Dieselben waren Thieren mit und ohne Icterus von 24 Stunden bis zu 7 Wochen nach der Vergiftung entnommen. Aber niemals konnte weder nach Alkoholhärtung noch Behandlung mit Sublimatlösung mit und ohne Anwendung der Fe-Reaction eine deutliche blutkörperchen- oder eisenhaltige Zelle in der Leber gefunden werden. Somit dürfte man wohl anzunehmen berechtigt sein, dass die weissen Blutkörperchen, wenigstens bei Winterschildkröten keine Rolle bei der Zersetzung des Hämoglobin spielen. Hiermit stimmt die Beobachtung überein, welche ich an Blutergüssen machte, die ich durch subcutane Injection von nicht defibrinirtem Schildkrötenblut erzeugte. Niemals sah ich, obgleich ich die Thiere mit dem eingespritzten Blut in einzelnen Fällen sehr lange leben liess $(17,30$ und 40 Tage), in diesem Blut blutkörperchenhaltige Zellen auftreten; niemals fand ich demgemäss einen Eisenreaction gebenden Körper oder Hämatoidin. Vielmehr wird bei Winterschildkröten das Hämoglobin aus den Blutkörperchen subcutaner Blutergüisse einfach allmählich ausgelaugt. Es wird durch diese Versnche die von $\mathrm{Naunyn}$ und Minkowski für Warmblïter aufgestellte Ansicht, dass zwar auch in den weissen Blutkörperchen eine Zersetzung des Hämoglobin stattfindet, der Hauptantheil aber den Leberzellen zufalle, bestätigt.

Bei der Anstellung der Fe-Reaction behufs Auffindung von eisenhaltigen Zellen war als constanter Befund eine mehr oder weniger starke Blaufärbung des ganzen Leberschnittes bemerkbar, die sich schon makroskopisch deutlich von auf gleiche Weise behandelten Leberschnitten normaler Schildkröten unterschied. Dieselbe war schon an der Leber von Schildkröten, die 24 Stunden nach der Vergiftung getödtet und bei denen eine vermehrte Gallenbildung noch nicht 
nachzuweisen war, bemerkbar. Sie war viel deutlicher bei Thieren, die später getödtet waren und schon Icterus oder eine grüne Verfärbung der Leber zeigten. Bei denjenigen, die erst nach 7 Wochen mit starkem Icterus zu Grunde gingen, konnte sie entweder gar nicht mehr oder nur sehr schwach nachgewiesen werden. Unter dem Mikroskop sah man bei schwacher Vergrösserung, dass die Blaufärbung auf umschriebene Stellen beschränkt war, indem theils Punkte, theils leicht geschlängelte Linien sich blaugefärbt von der Umgebung abhoben. Die Betrachtung bei starker Vergrösserung gab dann Aufschluss über ihre Lage. Wo eine Gallencapillare im Querschnitt getroffen war, sah man die sie begrenzenden Leberzellen in dem Theil, der sie unmittelbar umgab, blau gefärbt, während der dem Gallengang abgewendete Theil und der Kern farblos waren. Dies waren die bei schwacher Vergrösserung als Punkte wahrgenommenen Stellen, während die Längsstreifen Längsschnitten von Gallengängen entsprachen. Auch hier waren die ihn umgebenden Leberzellen in dem ihm zugewendeten Theil blau gefärbt, so dass der Gallengang von 2 blaugefärbten Streifen, die durch die aneinanderliegenden blauen Theile der Leberzellen entstanden waren, eingefasst war. Diese Blaufärbung war als feinkörnig zu erkennen. Ihre Intensität, unmittelbar am Gallengang am grössten, nahm nach dem Zellkern zu allmählich ab. Das Bild war sebr ähnlich dem von $\mathrm{Nauny}$ und Minkowski nach der Arsenwasserstoffvergiftung bei Gänsen beschriebenen. In Milz und Niere war niemals eine Spur von Eisen nachzuweisen.

Der constante Befund von Eisen in den Leberzellen bei den Thieren, die mit Arsenwasserstoff vergiftet waren, kann nur auf zwei Arten gedeutet werden. Entweder handelt es sich darum; dass das in die Blutbahn durch Lösung der rothen Butkörperchen ïbergegangene Hämoglobin in den Leberzellen zersetzt wird. Der Gallenfarbstoff, welcher nach Abspaltung des Eisens aus dem Hämoglobin hervorgeht, wird dann in die Gallengänge ausgeschieden. Das hierbei freiwerdende Eisen wird, wie Baserin's Versuche an Hunden zeigen, nicht ausgeschieden, sondern bleibt in den Leberzellen lange Zeit liegen. Die zweite Möglichkeit wäre die, dass das Eisen aus dem Blutfarbstoff bereits im Blut abgespalten und,als solches von den Leberzellen ans dem Blute aufgenommen wird. Diese Annahme ist nach Naunyn und Minkowski und namentlich nach Baserin's eben angeführten Beobachtungen sehr unwahrscheinlich. Doch wurde der Versuch gemacht, das noch weiter dadurch zu entscheiden, dass man nachsah, wie sich die Vertheilung des durch die Perl'sche 
Reaction nachweisbaren Eisens in den Organen nach Einführung löslicher Eisensalze ins Blut gestaltete. Stellten sich wesentliche Differenzen in der Vertheilung des Eisens auf die Organe nach der Arsenwasserstoffvergiftung einerseits und nach der Eiseneinführung andererseits heraus, so war es klar, dass bei ersterer das Eisen nicht schon frei im Blut circulirt, dass daher die Abspaltung desselben aus dem Blutfarbstoff nicht schon im Blut, sondern erst in der Leber statthat.

Von Zaleski ${ }^{1}$ ) ist die Anziehung und Ausscheidung des Eisens beim Warmblïter als eine specifische Function der Leber aufgefasst worden. Derselbe zog zwei Kaninchen eines Wurfes unter gleichen Bedingungen auf und spritzte dann dem einen ein lösliches Eisensalz in die Gefässe. Nach einigen Stunden wurden beide getödtet, ihre Organe vermittelst Durchspülung mit indifferenten Lösungen blutfrei gemacht und ihr Eisengehalt quantitativ bestimmt. Die Vergleichung des Eisengehalts der Organe des Thieres, dem Fe eingespritzt war, mit dem anderen nicht so behandelten ergab eine grössere Menge Eisen in der Leber des Thieres, dem dasselbe in das Blut gebracht war. Daraus den Schluss ziehen zu wollen, dass die Leber eine specifische Attraction für Eisen habe, scheint gewagt, da es auf Annahme beruht, dass ohne die Einspritzung von Eisen beide Lebern denselben Eisengehalt gehabt haben würden. Den Versuch, ob sich durch Erziehung von Thieren unter möglichst gleichen Bedingungen ein gleicher Eisengehalt in den entsprechenden Organen herstellen lässt, hat $\mathrm{Zaleski}$ nicht gemacht.

Die Befunde anderer Forscher stehen aber mit jener Annahme in directem Widerspruch. So konnten Gläveke ${ }^{2}$ ) und Kobert ${ }^{3}$ ) nach Einfiihrung von Eisensalz ins Blut dasselbe reichlich in der Niere nachweisen. Besonders gut gelingt es, durch Injection von Eisensalzen bei Schildkröten die Vertheilung desselben im Körper festzustellen, da ihre Organe normalerweise keine Fe-Reaction geben, und es zeigt sich, dass hierbei eine ganz andere Vertheilung des Eisens im Körper wie nach der Arsenwasserstoffvergiftung zu Stande kommt; es ist danach sehr unwahrscheinlich, dass der eisenhaltige Körper bei $\mathrm{As}_{3}-$ Vergiftung als soleher im Blut eirculirt hat.

Die Menge der subcutan einzuspritzenden Eisensalze darf nur gering sein, da das Eisen sehr giftig wirkt. Wir nahmen, um die-

1) Zur Frage über die Ausscheidung des Eisens aus dem Thierkörper und zur Frage der Menge dieses Metalls bei hungernden Thieren. Dies. Arch. XXIII. Bd.

2) Deber subcutane Eiseninjection. Ebenda. XVII. Bd.

3) Zur Pharmakologie des Eisens und Mangans. Ebenda. XVI. Bd. 
jenigen Mengen von Eisen einzuführen, welche nicht viel grösser sind, als diejenigen, welche möglicherweise auch aus dem Hämoglobin bei der Arsenwasserstoffvergiftung abgespalten werden könnten, folgende Schätzung an. Das gesammte Blut einer Schildkröte von 60 bis $70 \mathrm{~g}$ Gewicht mag ungefähr $0,5 \mathrm{~g}$ Hämoglobin und somit etwa $3 \mathrm{mg}$ Eisen enthalten. Bei der Arsenwasserstoffvergiftung der Schildkröten scheint oft schnell der grösste Theil der Blutkörperchen in einem Tage zerstört zu werden. Da nach 24 Stunden bereits Icterus auftritt, so muss in der Zeit bereits ein sehr erheblicher Theil des Hämoglobin zersetzt und sein Eisen abgespalten sein. Ich schätzte die Menge des so in den nächsten Tagen in die Circulation gelangenden Eisens auf $1 / 2-1 \mathrm{mg}$, eine Schätzung, die meiner Ansicht nach nicht zu hoch ist. Um ähnliche Bedingungen durch Injection von Eisensalzen herzustellen, wurden solch geringe Dosen Ferrum pyrophosphoricum cum Natro citrico subcutan injicirt, so dass das Salz nur langsam in die Circulation gelangt.

Nach subcutaner Injection der kleinsten Dosis Eisen, welche überhaupt noch $\mathrm{zu}$ einer durch mikrochemische Reaction nachweisbaren Eisenablagerung in den Organen führt, d. h. nach einmaliger Injection von $1 / 2 \mathrm{mg}$, oder nach an zwei auf einander folgenden Tagen wiederholten Injectionen von $1 / 4 \mathrm{mg}$ Eisen in Form des genannten Salzes ${ }^{1)}$ gaben 24 Stunden danach die Leberzellen meist gar keine Eisenreaction; nur in ganz vereinzelten Fällen fand ich schon in den ersten Tagen nach der Injection nach Anwendung von Ferrocyankalium und Salzsäure eine Blaufärbung der. Leberzellen in einzelnen Acini. Dieselbe war selbst bei grösseren Dosen immer gering und meist erkannte man deutlich, dass es sich hier um Ausscheidung des Eisens in der Galle handle; denn es lagen die blaugefärbten Massen zum Theil unzweifelhaft in den Gallencapillaren zwischen den Leberzellen. Hingegen nahmen die grösseren Lebergefässe und ab und $\mathrm{zu}$ eine Capillare leicht blaue Färbung an. Auch in der Milz zeigten zahlreiche Gefässränder leichte Reaction, d. h. deutliche blaue Färbung. In der Niere war nur hin und wieder an einem Glomerulus eine leichte Spur von Blaufärbung wahrnehmbar. Diese Bilder wurden viel deutlicher, wenn grössere Eisenmengen auf einmal (bis za. $1 \mathrm{cg}$ ) oder kleine Dosen $(1 \mathrm{mg})$ einige Tage hinter einander bis zum Gesammtbetrage von 3-4 mg injicirt waren. Die Wände der

1) $1 \mathrm{mg}$ Eisen ist in $5 \mathrm{mg}$ Ferrum pyrophosphoricum cum Natro citrico enthalten. Die hier angegebenen Dosen geben immer die Menge des Eisens, welches in den eingespritzten Dosen von Ferrum pyrophosphoricum cum Natro citrico enthalten war. 
grösseren Lebergfässe wurden dann stark blau. Auch die Capillaren traten durch ihre Blaufärbung bei der Reaction schön hervor und umschlossen netzförmig die Leberzellen. Letztere blieben entweder ganz ungefärbt, oder es traten feine blaue Körnchen hervor, über das Protoplasma der ganzen Zelle vertheilt und ohne die Anordnung an dem dem Gallengang zugekehrten Pol der Zelle, wie sie fur die Arsenwasserstoffvergiftung beschrieben ist. Mitunter fiel es auf, dass gerade der Kern und seine nächste Umgebung stark gefärbt waren. Die in den Gefässen enthaltenen rothen Blutkörperchen waren unverändert. Hingegen hatten die weissen Blutkörperchen Eisen aufgenommen und lagen als blaue Kügelehen zahlreich in den Capillaren, während sie in den grösseren Blutgefässen, umgeben von rothen Blutkörperchen, seltener zu bemerken waren. Jedoch habe ich sie auch einige Male in den grösseren Gefässen und besonders deutlich in Nierengefässen gefunden. Interessant gestaltet sich die Eisenvertheilung in der Milz. Das Parenchym derselben gab gar keine Eisenreaction; nur die Gefâsswände, diese aber sebr stark, batten das Eisen aufgenommen. Obgleich dann alle Gefässhänte sich blau färbten, so war doch die Färbung am intensivsten an den Endotbelien; dies war auf Querschnitten der Gefässe sicher zu erkennen.

In der Niere waren weitaus am meisten die Glomeruli mit Eisen gefüllt. Nach der Einführung geringer Eisenmengen waren nur kleine Stellen derselben blau, nach den grössten schliesslich das ganze Convolut ihrer Gefässchen. $A b$ und zu hatten auch ein Vas afferens und andere Gefässe der Niere Eisen anfgenommen, niemals aber fand ich es in den Epithelien der Harnkanälchen.

Dieser letztere Befund ist verschieden von dem, den Gläveke und Kobert nach Eiseninjection erboben haben. Bei diesen lag, wie ich nach einigen Versuchen bei Kaninchen und Hunden nur bestätigen kann, das ausgeschiedene Eisen hauptsächlich in den Epithelien der Harnkanälchen, obgleich die Glomeruli durchaus nicht frei davon waren.

Eine ganz andere und, um es gleich hier zu bemerken, nach der Arsenwasserstoffvergiftung vorkommende gleiche Vertheilung, des Eisens in den Organen' zeigte sich bei Thieren, welche 3-4 Tage nach einmaliger Injection mehrerer Milligramme Eisen - wieder in der Form des Ferrum pyrophosphoricum cum Natro citrico - gestorben oder getödtet waren. Milz und Niere gaben jetzt gar keine Eisenreaction mehr; dagegen fand man in der Leber ganz dasselbe Bild, wie es oben für die Arsenwasserstoffvergiftung beschrieben ist. Es lag nahe, anzunehmen, dass es sich hier nicht mehr um die einfache 
Ablagerung des eingeführten Eisens, sondern um die Folgen der Eisenvergiftung handle. Dementsprechend ergab die Untersuchung des Blutes dieser Thiere, ziemlich reichlich ihres Hämoglobins beraubte rothe Blutkörperchen, die durch ihren Kern und das deutlich sichtbare, wenn auch vollkommen farblose Stroma als solche kenntlich waren (Schatten). Auch zeigten rothe Blutkörperchen Vacuolenbildung; die Vacuolen, öfters von der Grösse des Kernes, mitunter auch viel kleiner, imponirten als runde Lücken mit stark lichtbrechenden Rändern im Stroma. Daher dürfte das Eisen in grossen Dosen wohl dieselbe zerstörende Wirkung auf die rothen Blutkörperchen wie Arsenwasserstoff haben.

Hier haben wir also nicht mehr das eingefiuhrte Eisen vor uns, sondern müssen annehmen, dass das Auftreten des Eisens mehrere Tage nach der Injection von Ferrum pyrophosphoricum eum Natro citrico ebenso, wie nach der Arsenwasserstoffvergiftung, auf eine Verarbeitung des Hämoglobins in den Leberzellen zu beziehen ist, die Verarbeitung des Blutfarbstoffs nämlich, welcher durch die Eisenvergiftung aus den rothen Blutkörperchen ins Blutserum gelangt.

Im Blute circulirende Eisensalze lagern sich also bei Winterschildkröten in den verschiedenen Organen ab. Eine besondere Vorliebe scheinen sie nur für die Gefässwände, besonders die der Milz, und die weissen Blutkörperchen zu haben. Wo aber das Eisen in die Leberzellen übergeht, was ja für den Theil desselben, der mit der Galle ausgeschieden wird, selbstverständlich ist, nimmt es niemals die charakteristische Lagerung an, wie sie ron den eisenhaltigen Körpern in den Leberzellen nach der Arsenwasserstoffvergiftung beschrieben ist.

Mit Hinzuziehung dieser Beobachtungen darf man aus dem Fehlen jeglicher Eisenreaction in der Niere und der Milz das Auftreten derselben in den Leberzellen bei Thieren, die mit Arsenwasserstoff vergiftet waren, mit grosser Wahrscheinlichkeit darauf beziehen, dass bei der Arsenwasserstoffrergiftung die Abspaltung des Eisens aus dem Hämoglobin nicht im Blut statthat, sondern erst in den Leberzellen. Hier wird das Hämoglobin erst zersetzt, das Eisen abgespalten und aus dem Reste Gallenfarbstoff gebildet. Letzterer wird mit der Galle ausgeschieden. Das Eisen, welches bei seiner Bildung aus Hämoglobin als Nebenproduct abfällt, wird nicht in der Galle ausgeschieden, wie Baserin nachgewiesen hat. Er erzengte bei Hunden durch Vergiftang mit Arsenwasserstoff eine vermebrte Gallenfarbstoff bildung und fand den Eisengehalt der Galle nicht vermehrt. Was aber aus dem so in den Leberzellen nach der Arsenwasserstoff- 
vergiftung durch die Ferrocyankalium-Salzsäurereaction nachweisbaren Eisen wird, ist noch völlig unklar.

Das Resultat dieser Untersuchung ist kurz zusammengefasst folgendes:

1. Es gelingt bei Winterschildkröten durch Vergiftung mit Arsenwasserstoff Icterus zu erzeugen.

2. In den Leberzellen solcher Thiere findet man einen eisenhaltigen Körper, der auf eine in den Zellen stattfindende Zersetzung des Hämoglobins mit gleichzeitiger Büldung von Gallenfarbstoff bezogen werden muss.

3. Die weissen Blutkörperchen, die nach $\mathrm{Naunyn}$ und $\mathrm{Min-}$ kowski bei Vögeln an der Gallenfarbstoffbildung einen geringen Antheil haben, spielen bei Winterschildkröten bei derselben keine Rolle.

Februar 1888. 